\title{
Rapid Publication and Announcement of the 2014 JCS Meeting Activities
}

Hiroaki Shimokawa, MD, PhD

Dear Colleagues,

The $78^{\text {th }}$ Annual Scientifc Meeting of the Japanese Circulation Society (JCS 2014) will be held in Tokyo on March 21-23, 2014 (Congress Chairperson, Ryozo Nagai, MD, PhD). The Circulation Journal rapidly publishes and announces JCS 2014 activities as follows:

\section{Late Breaking Clinical Trials / Late Breaking Cohort Studies Papers}

Papers selected for presentation in the Late Breaking Clinical Trials (LBCT) / Late Breaking Cohort Studies (LBCS) sessions in JCS 2014 will be rapidly published, after peer review, in the April issue of the Journal. In addition, these LBCT / LBCS papers (JCS 2014) will be published online along the presentation in order to attract widespread attention of the attendees, as well as readers worldwide.

\section{JCS 2013 Awards}

We are pleased to announce the following JCS Awards to be presented during the JCS 2014 meeting:

\section{Sato Award by the Japan Heart Foundation and the JCS (39 $\left.{ }^{\text {th }}\right)$}

Tatsuya Shimizu, MD, PhD

(Institute of Advanced Biomedical Engineering and Science, Tokyo Women's Medical University)

"Cell Sheet-Based Tissue Engineering for Heart Regeneration"

2. CPIS (Cardiovascular Pharmacotherapy International Symposium) Award (22 ${ }^{\text {nd }}$ )

Takeya Minami, MD, PhD

(Department of Cardiovascular Medicine, Kyoto University Graduate School of Medicine)

"Reciprocal Expression of MRTF-A and Myocardin Is Crucial for Pathological Vascular Remodelling in Mice"

\section{Cardiovascular Surgery Award $\left(9^{\text {th }}\right)$}

Masashi Kawamura, MD, PhD

(Department of Cardiovascular Surgery, Osaka University Graduate School of Medicine)

"Enhanced Survival of Transplanted Human iPS Cell-Derived Cardiomyocytes by the Combination of Cell-Sheets With the Pedicled Omental Flap Technique in a Porcine Heart"
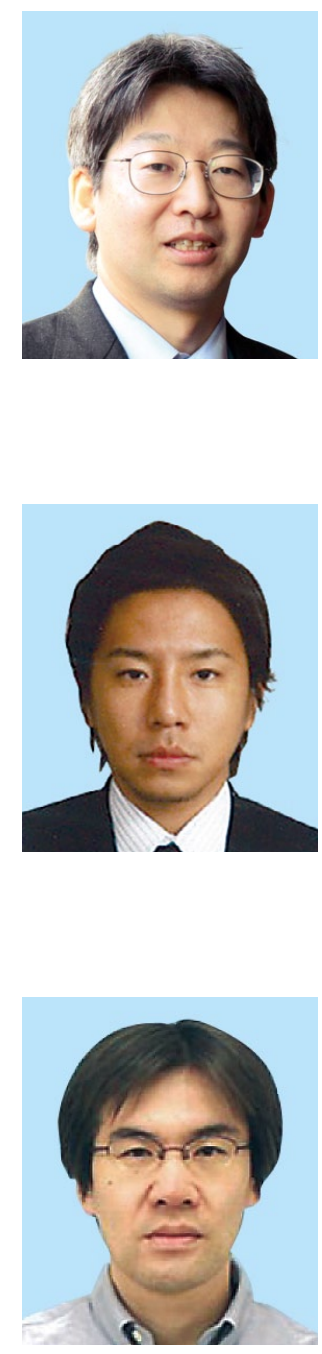


\section{Pediatric Cardiology Award $\left(9^{\text {th }}\right)$}

Hironori Matsuhisa, MD, PhD

(Department of Cardiovascular Surgery, Kobe Children's Hospital)

"Ventricular Septal Dysfunction After Surgical Closure of Multiple Ventricular Septal Defects"

\section{Woman Researcher's Award ( $7^{\text {th }}$ )}

Yohko Yoshida, MD, PhD

(Department of Cardiovascular Biology and Medicine, Niigata University Graduate School of Medical and Dental Sciences)

"Semaphorin3E-Induced Inflammation Contributes to Insulin Resistance in Dietary Obesity"

\section{Cardiovascular Clinical Research Investigator's Award (3 $\left.{ }^{\text {rd }}\right)$}

\section{Clinical Research Investigator}

Hiroshi Watanabe, MD, PhD

(Department of Cardiovascular Biology and Medicine, Niigata University Graduate School of Medical and Dental Sciences)

"Identification of Causative Genes and Mechanisms, and Development of Novel Therapy in Arrhythmia Syndromes"

\section{Case Report}

\section{[First Place]}

Hiro Yamasaki, MD, PhD

(Cardiovascular Division, Faculty of Medicine, Tsukuba University Hospital)

"Ischemic Esophageal Ulceration That Developed After an Early Endoscopic Surveillance in a Patient Receiving Catheter Ablation for Atrial Fibrillation"

[Second Place] (in alphabetical order)

Mike Saji, MD

(Advanced Cardiac Valve Center, Department of Medicine, University of Virginia)

"Autopsy Finding of the Sapien XT Valve From a Patient Who Died Suddenly After Transcatheter Aortic Valve Replacement"

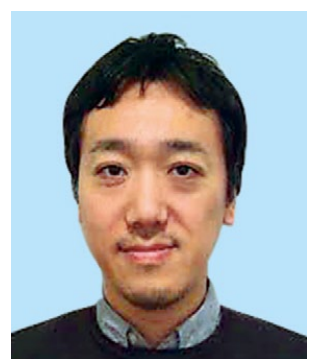


Hiromichi Wakui, MD, PhD

(Department of Medical Science and Cardiorenal Medicine, Yokohama City University Graduate School of Medicine)

"Endovascular Treatment of Renal Artery Stenosis Improves Contralateral Renal Hypertrophy With Nephrotic Syndrome"

\section{Cardiovascular Imaging Award ( $\left.1^{\text {st }}\right)$}

\section{[First Place]}

Toshiro Kitagawa, MD, PhD

(Department of Cardiovascular Medicine, Hiroshima University Graduate School of Biomedical and Health Sciences)

"Integrin-Targeted Molecular Imaging of Experimental Abdominal Aortic Aneurysms by ${ }^{18}$ F-FPPRGD 2 Positron Emission Tomography"

[Second Place] (in alphabetical order)

Masae Uehara, MD, PhD

(Department of Cardiovascular Medicine, Chiba University Graduate School of Medicine and Department of Cardiovascular Medicine, The University of Tokyo Hospital)

"Diagnostic Accuracy of 320-Slice Computed-Tomography for Detection of Significant Coronary Artery Stenosis in Patients With Various Heart Rates and Heart Rhythms Compared With Conventional Coronary-Angiography"

Kihei Yoneyama, MD, PhD

(Cardiology, Department of Internal Medicine, St. Marianna University School of Medicine)

"Age, Sex, and Hypertension-Related Remodeling Influences Left Ventricular Torsion Assessed by Tagged Cardiac Magnetic Resonance in Asymptomatic Individuals: The MultiEthnic Study of Atherosclerosis"
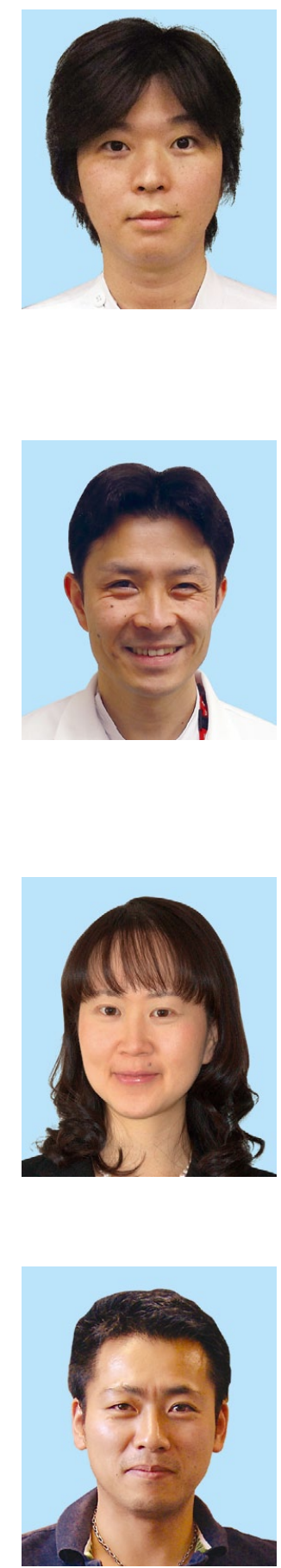

The final results of the Young Investigator Awards (YIA) Competition, those of the International YIA Competition and those of Comedical Awards will be announced in the next issue of the Journal.

Hiroaki Shimokawa, MD, $\mathrm{PhD}$

Editor-in-Chief

Circulation Journal

(Released online March 14, 2014) 